\title{
IL RUOLO DELLE CITTÀ PORTUALI NELLE DINAMICHE DEL COMMERCIO TRA ADRIATICO E IONIO NELL'ALTO MEDIOEVO (V-IX SEC.)
}

\section{ENRICO CIRELLI}

UDC: $339.165 \cdot 4(262) " 04 / 08 "$

Review $711.453 \cdot 4(262) " 04 / 08 "$

Manuscript received: 27. 12. 2015.

Revised manuscript accepted: 10. 03. 2016.

DOI: 10.1484/J.HAM.5.111329
E. Cirelli

Alexander von Humboldt Stiftung - Alma Mater Studiorum Università di Bologna Bologna, Italia enricocirelli@hotmail.com

Between Late Antiquity and the Early Middle Ages the routes that connected East and West underwent many changes. New archaeological discoveries show the economic vitality of some Ionian port cities close Corfu's channel during the $7^{\text {th }}$ and $8^{\text {th }}$ century, in contrast to other Mediterranean regions. Some pottery contexts found in the ports of Saranda, Butrint and Durres are framed perfectly in the dynamics of Mediterranean trade, with substantial growth of quantity of finds especially at this time. This trend is very different from what occurred in Middle and North Adriatic sites. The numerous contexts so far studied a spike in imports towards the middle of the fifth century and a consistent volume of trade until the middle of the G $\sigma^{\text {th }}$ century. In this period we observed in the Adriatic port-cities a turnaround of imports from North Africa, towards the Eastern Mediterranean products, that Justinian war was not only a military conquest but also the supremacy of Eastern merchants in Western ports.

Keywords: Trade, cities, ports, Adriatic, routes

\section{INTRODUZIONE}

I percorsi viari che collegavano Oriente e Occidente subirono numerosi cambiamenti tra la tarda Antichità e i primi secoli dell'alto Medioevo'. Soprattutto tra VI e VIII secolo i viaggi via terra non dovettero essere particolarmente agevoli. È stato già abbondantemente dimostrato che diminuirono di numero e aumentarono di $\operatorname{costo}^{2}$. Il più grande cambiamento che avvenne in questo periodo fu probabilmente lo smembramento dei due corridoi che mettevano in comunicazione Europa centrale e occidentale con Costantinopoli el'Oriente, con un riassetto forse databile solo all'età carolingia ${ }^{3}$.

Uomini e merci avevano viaggiato lungo questi complessi di frontiera costituiti dal Danubio per diversi secoli. Quando nel VI secolo tale frontiera cadde sotto assedio, prima gli Avari, poi i Bulgari occuparono le regioni del medio e basso corso; venne meno il controllo di Costantinopoli per più di quattro secoli ${ }^{4}$. Quale sia stato il ruolo degli insediamenti urbani costieri, in queste trasformazioni, è l'obiettivo principale di questo contributo.

Negli ultimi decenni l'archeologia ha infatti consentito di declinare in maniera molto più articolata il dibattito sulle trasformazioni dei centri urbani. L'abbandono di alcune città di fondazione romana in diverse province del Mediterraneo e il nuovo assetto dei territori sono molto diversi da regione a regione e le tendenze non sono sempre simili. La sopravvivenza e la continuità d'uso di molti centri dipende spesso dalla vitalità economica e dalla possibilità di gestire un territorio. Favorite dalla loro posizione, la maggior parte delle città costiere continuarono a essere utilizzate. $\mathrm{Si}$ formarono inoltre alcuni insediamenti con caratteristiche protourbane, grazie alla loro centralità negli scambi e grazie alla loro posizione lungo perocorsi fluviali o all'interno di importanti insenature portuali. La nascita e il declino della città tardoantica di Classe costituiscono un importante esempio ${ }^{5}$ e in area altoadriatica la tenuta dei centri urbani e la fioritura di nuovi insediamenti nei secoli successivi è stata più volte dimostrata ${ }^{6}$.

\section{I SEGNI DELLA CRISI}

In Romagna e nell'Adriatico centrale sono stati evidenziati fenomeni di crisi generale delle città e del territorio già nel corso del IV secolo, fino alla ripresa e al nuovo assetto economico che si è stabilito agli inizi del secolo successivo7. Questa regione che nei secoli precedenti aveva prodotto ed esportato vino di grande qualità, realizzato soprattutto nel territorio di Forlimpopoli e nella zona di Santarcangelo di Romagna, entra in crisi su molti livelli. A Galeata, nell'entroterra forlivese, gli scavi più recenti hanno permesso di

\footnotetext{
${ }^{1}$ Questo contributo è stato scritto nell'ambito del progetto CROMART, Croatian medieval heritage in European context: mobility of artists and transfer of forms, functions and ideas, diretto dal prof. Miljenko Jurković e di cui faccio parte, grazie anche al sostegno della Fondazione Alexander von Humboldt e Carl Friefrich von Siemens, per cui svolgo un incarico di ricerca avanzata presso la LMU, di Monaco di Baviera.

${ }^{2}$ M. MCCORMICK Origins of the European Economy. Communications and Commerce, A.D. 300-90o, Cambridge, 2001, p. 64.

3 D. OBOLENSKI, The Balkans in the ninth century: barrier or bridge?, in Byzantinische Forschungen 13, 1998, p. 47-66, part. p. 53-59.

${ }^{4}$ M. MCCORMICK, op cit. (n. 1); N.G.L. HAMMOND A History of Macedonia, Oxford, 1972, pp. 19-58

${ }^{5}$ A. AUGENTI, Città e porti dall'Antihità al Medioevo, Roma, 2010; ID., Classe, Archaeologies of a Lost City, in N. Christie, A. Augenti (eds.), Urbes Extinctae. Archaeologies of Abandoned Classical Towns, London, 2012, p. 45-75, part. p. 67.

${ }^{6}$ S. GELICHI, Flourishing places in North Eastern Italy: towns and emporia between late Antiquity and the Carolingian Age, in J. Henning (ed.), Post Roman Towns. Trade and settlement in Europe and Byzantium: the heirs of the Roman West, Berlin, 2007, p. 77-104.

7 V. MANZELLI, Ravenna, 2000, p. 236.
} 
trovare i resti di un complesso produttivo piuttosto esteso, con alcune fornaci defunzionalizzate improvvisamente tra III e IV secolo d.C. ${ }^{8}$ Evidenze di distruzione e abbandono si riconoscono anche nel territorio a sud di Ravenna, nell'agro decimano ${ }^{9}$, nella villa di Russi ${ }^{10}$, nell'entroterra ravennate e più a sud a San Pietro in Cotto nella Romagna meridionale e nel territorio di Rimini più in generale ${ }^{11}$. Anche in Abruzzo gli insediamenti più importanti del territorio nella media età imperiale conoscono una fase di crisi produttiva ${ }^{12}$. Non sono molte le ceramiche prodotte localmente, ma tra i contenitori da trasporto sono note anche in questo caso alcune anfore a fondo piatto la cui fabbricazione finisce nella prima metà del III sec. d.C.

\section{LA RIPRESA DEL V SECOLO}

La ripresa si ha con la trasformazione delle infrastrutture portuali che si attrezzano per attrarre e redistribuire in Italia merci destinate al mercato dell'Italia centro-settentrionale e l'Europa subalpina attraverso i circuiti padani. La città di Ravenna è il volano di questa rinascita economica e grazie all'attività commerciale d'intermediazione molti prodotti del Mediterraneo orientale e della Tunisia raggiungono l'intera regione e in seguito l'Italia centro settentrionale. Oltre alle vaste importazioni di anfore provenienti da ogni porto del Mediterraneo e di vasellame fine da mensa in larga parte tunisino, sono attestate produzioni regionali di grande pregio, destinate a un mercato piuttosto vasto, anche se soprattutto destinato all'Italia centrale.

La prosperità economica di questo periodo si diffonde in tutto il territorio e raggiunge sia le città dell'asse emiliano sia i piccoli insediamenti rurali ${ }^{13}$. Sono infatti testimoniati nuovi investimenti delle aristocrazie oltre che nelle città principali della regione, anche all'interno di ville prestigiose e in altri tipi di insediamento, come ad esempio la statio di Cesenatico, a lato della via Popilia, dove recenti scavi mostrano un'esplosione di monete databile tra IV e V sec. ${ }^{14}$.

Ancora più interessanti per le forme di popolamento $i$ nuovi agglomerati produttivi organizzati attorno ad alcune fornaci tardoantiche, lontani da centri urbani e vicine solo agli assi viari e agli approdi endolagunari, come nel caso di Santarcangelo ${ }^{15}$.

Sealcune ville, soprattutto quelle legate alla corte imperiale, diventano centri polarizzatori e dopo il VI secolo decadono completamente o vengono abbandonati, vengono anche creati nuovi siti, come San Zaccaria che nasce in età tardoantica su un'area scarsamente frequentata in età romana ${ }^{16}$.

Oltre alle ceramiche disponiamo di molte altre evidenze archeologiche che consentono di affrontare queste valutazioni: dalle grandi quantità di marmi preziosi ${ }^{17}$, alle pregevoli produzioni in vetro, fino alla circolazione monetaria e agli aspetti economici più legati alla fiscalità.

Nelle Marche il quadro è invece piuttosto diverso ${ }^{18}$. Anche se è sintomatica l'assenza di anfore tardoantiche di produzione locale. Sono comunque presenti alcune anfore di importazione, necessarie per far arrivare olio e vino attraverso l'adriatico, ma in quantità decisamente inferiore. In questo caso gli studiosi sostengono una diversa modalità di circolazione dei prodotti con un mercato che dipende soprattutto dall'esterno piuttosto che dalla coltivazione diretta. Ad esempio si potrebbe pensare a un aumento del consumo interno che rendeva inutile il trasporto in anfora.

Molti dati editi dell'archeologia marchigiana vengono tuttavia da lunghi progetti di studio su siti abbandonati, che dunque restituiscono poche informazioni per l'alto Medioevo. Al contrario l'archeologia d'emergenza nei siti a continuità di vita, che indaga i periodi che qui ci interessano sono ignorate. Nuovi e importanti dati vengono dal sito di Senigallia, dagli scavi dell'area archeologica 'La Fenice', dove lo studio della ceramica ha mostrato una notevole vitalità del centro anche durante il periodo di occupazione ostrogota ${ }^{19}$.

Più a sud, nell'insediamento portuale di Pescara tra V e VI sec. d.C. si nota un aumento di importazioni di ceramica africana. In questo periodo va anche sottolineata la nascita di una nuova produzione ceramica, legata a influenze culturali orientali, come sostenuto da Staffa, o forse a un nuovo impulso produttivo regionale, come discusso di recente ${ }^{20}$.

\footnotetext{
${ }_{8}^{8}$ R. VILLICICH, Scavi nell'area della villa di Teoderico a Galeata: $i$ nuovi dati, in FoldEr The Journal of Fasti Online 261, 2012 , p. 1-13.

9 N. MANCASSOLA, Le forme del popolamento rurale nel territorio Decimano dalla caduta dell'Impero Romano all'anno Mille, in M. Ficara, V. Manzelli (eds.), Orme nei Campi. Archeologia a sud di Ravenna, Firenze, 2008, p. 89-103.

${ }^{10}$ E. BALDINI, D. BOLOGNESE (eds.), Storia di Russi. Dalla villa alla città, Ravenna, 2014, p. 335-346.

"S. SANTORO, Persistenza, continuità e discontinuita' fra romanita' e medioevo nei centri di culto extraurbani ai confini fra Romagna e Marche, in Atti del VI Convegno Nazionale di Archeologia Cristiana, Pesaro, 1983, p. 239-250.

${ }^{12}$ A. STAFFA, Considerazioni su produzioni ceramiche e commerci nell'Abruzzo Adriatico fra VI e VIII secolo, in E. Cirelli, F. Diosono, H. Patterson (eds.), Le forme della crisi. Produzioni ceramiche e commerci nell'Italia centrale tra Romani e Longobardi. Atti del convegno internazionale (Spoleto, Campiello sul Clitunno 2012), Bologna, 2015, p. 593-616.

${ }^{13}$ M. LIBRENTI, Ricognizione di superficie e insediamento medievale nella pianura emiliano romagnola: alcune considerazioni, in G.P. Brogiolo (ed.), Atti del II Congresso Nazionale di Archeologia Medievale, Firenze, 2000, p. 170-174.

${ }^{14}$ D. SAMI et al., Ad Novas-Cesenatico. From Roman Road to Late Antique Wooden Structures. An Interim Report on the Evaluation Test Pits and Excavation at Cà Bufalini, 2006, in FoldEr The Journal of Fasti online 318, 2014, p. 1-20.

${ }^{15}$ M.L. STOPPIONI, Romagna sud-orientale e appenninica: imitazioni fittili di ceramiche da mensa e di stoviglie metalliche da portata nella tarda Antichità, in Le forme della crisi, op. cit. (n. 10), p. 63-74.

${ }^{16}$ M. CAVALAZZI, M. FICARA, Importazioni e cultura materiale in età tardoantica nell'Ager Decimanus, in Le forme della crisi, op. cit. (n. 10$)$, p. 53-62.

${ }^{17}$ I. BALDINI et al., Manufatti marmorei a Ravenna in età tardoantica: produzioni e committenza, in E. Cirelli, E. Giorgi, G. Lepore (eds.), Economia e territorio nell'Adriatico centrale tra tarda Antichità e alto Medioevo (IV-VIII sec.), Atti del Convegno internazionale (Ravenna, 2014), Bologna, fc.

${ }^{18}$ A. GAMBERINI, Contenitori da trasporto e commerci nelle Marche in età tardoantica, in E. Cirelli, F. Diosono, H. Patterson, op. cit. (n. 10), p. $239-252$.

${ }^{19}$ I materiali sono ancora in corso di studio da parte di chi scrive grazie a un accordo tra Soprintendenza per i Beni archeologici delle Marche, Università di Bologna e Comune di Senigallia, sotto la direzione del prof. G. Lepore.

${ }^{20}$ A. DE IURE, La diffusione della ceramica dipinta a bande in Abruzzo tra tarda Antichità e alto Medioevo, in Le forme della crisi, op. cit. (n. 10), p. 625-63o.
} 
Sta di fatto che questa produzione ceramica, la cosiddetta ceramica di tipo Crecchio, viene anche esportata in varie direzioni, fino a raggiungere Classe e verso sud il Molise ${ }^{21}$. Alcuni esemplari sono anche segnalati sulle coste croate, vicino Split e nell'isola di Sv. Klement a poca distanza ${ }^{22}$. Forse questo si spiega pensando al porto di Pescara come terminale per Spalato e Salona ${ }^{23}$.

Un grande cambiamento, lungo le coste dell'Adriatico, si osserva nella seconda metà del VI secolo. Il dato piàù rilevante è l'inversione dell'asse delle importazioni dall'Africa all'Oriente in gran parte dei siti che si affacciano sull'Adriatico, a dimostrazione che la conquista di questi territori significò anche una conquista di mercato, così come dimostrato per altre regioni del Mediterraneo ${ }^{24}$. Tra VI e VII, inoltre, il complesso dei ritrovamenti ceramici provenienti dall'area calabrese o siciliana risulta piuttosto esiguo lungo le coste adriatiche, sul versante italiano, e verosimilmente su quello balcanico. Per quanto riguarda i contenitori da trasporto sono presenti anfore vinarie di produzione calabrese del tipo Keay LII, ma rappresentano solo scarse percentuali del totale dei reperti anforacei tardoantici ( $4 \%$ a Classe).

Classe nel VII secolo è probabilmente uno dei centri di produzione di lucerne tipo Provoost 10, dette anche "lucerne siciliane" (dalla diffusione della loro produzione sull'isola) o "a rosario". L'Italia meridionale, dunque, in base alla letteratura archeologica disponibile, sembra entrare solo marginalmente nel flusso dei traffici di importazione diretti verso il porto adriatico.

Il VII secolo segna un ulteriore momento di cambiamento rispetto ai secoli precedenti. Già durante il governo di Eraclio si nota una notevole riduzione delle importazioni, anche se queste non si interrompono del tutto. Lungo la maggior parte dei siti adriatici sono distribuite, anche se in misura notevolmente inferiore, rispetto al secolo precedente, anfore orientali e vasellame fine da mensa, sia africane sia orientali insieme a vasellame da cucina ${ }^{25}$. La distribuzione di questo commercio è affidato ad altri attori che non hanno la medesima capacità imprenditoriale dell'enorme apparato statale, anche di quello tardoantico.
Insieme a queste importazioni viaggiano merci di ogni genere, e questo flusso non subisce alcuna interruzione anche dopo la conquista araba, ma solo una decrescita nel volume delle attestazioni. Oltre ad alcuni graffiti rinvenuti su anfore orientali della seconda metà del VII secolo ${ }^{26}$, che dimostrano la presenza di mercanti e prodotti di origine islamica, tra cui certamente il vino, vi sono altri numerosi indicatori della circolazione di uomini e l'interscambio su vari livelli con il mondo orientale anche per quel che riguarda la produzione artistica lungo i circuiti padani e nordadriati$\mathrm{Ci}^{27}$. Il numero di questi scambi sembra aumentare verso la fine dell'VIII e nel IX secolo, con l'evidenza di nuove anfore importate dall'Est ${ }^{28}$.

Nel versante opposto dell'Adriatico e verso quelle che sono considerate le porte di quello che viene chiamato 'il mare dell'intimità', le indagini archeologiche degli ultimi 15 anni hanno potuto dimostrare un'importante crescita del dinamismo economico e di investimenti delle artistocrazie nella costruzione di ricchi edifici e di fastose residenze, sia nei centri urbani sia nel territorio. Si tratta delle città legate al percorso che partendo dal porto di Patrasso attraversava il canale di Corfù, facendo scalo nelle città costiere di Butrinto e Onkesmos\Saranda, per poi lasciare le coste balcaniche e raggiungere il canale di Taranto o per risalire verso nord sia lungo la costa italiana o quella orientale balcanica, a seconda del periodo dell'anno con il favore delle correnti di risalita verso le città e gli emporia adriatici' ${ }^{29}$ (fig. 1).

La predilezione di questo percorso, più costoso ed estremamente più lungo rispetto a quello terrestre, dipese in parte dalla colonizzazione slava ${ }^{30}$. Basti pensare al viaggio compiuto dall'imperatore Costante, nel 662, che al seguito di un vasto esercito e insieme alla sua corte, si imbarcò in un periplo dell'Ellade per raggiungere la Sicilia. Nel tratto fino ad Atene il percorso fu svolto seguendo le regioni costiere "per litoraria Athenas" ed evitando la via Egnatia e il 'comodo' e tradizionale passaggio da Durazzo a Brindisi ${ }^{31}$. Misterioso il resto del tragitto, forse interamente via mare fino a Taranto, che raggiunse nel 663 , lasciando le coste ioniche solo all'altezza di Onchesmos.

\footnotetext{
${ }^{21}$ V. CEGLIA, I. MARCHETTA, Dinamica degli scambi tra la costa e l'entroterra molisano alla luce di vecchie e nuove acquisizioni (fine V-VII secolo), in Le forme della crisi, op. cit. (n. 10), p. 647-662.

${ }^{22}$ Ringrazio Marina Ugarkovic per la segnalazione.

${ }^{23}$ A. STAFFA, Insediamento e circolazione nelle regioni adriatiche nell'Italia centrale fra VI e IX secolo, in G.P. Brogiolo, P. Delogu (eds.), L’Adriatico dalla tarda Antichità all'età carolingia, Atti del convegno di studi (Brescia, 2001), Firenze, 2005, p. 109-182.

${ }^{24}$ S. FONTANA, Un "immondezzaio" di VI secolo da Meninx: la fine della produzione della porpora e la cultura materiale a Gerba nella prima età bizantina, in M. Khanoussi, P. Ruggeri, L’Africa Romana. Geografi, viaggiatori, militari nel Maghreb: alle origini dell'archeologia nel Nord Africa, Atti del XIII Convegno Internazionale di Studi (Djerba, 1998), Roma, p. 95-114.

${ }_{25}$ P. ARTHUR, Pots and boundaries. On cultural and economic areas between Late Antiquity and the Early middle Ages, in M. Bonifay, J.C. Tréglia (eds.), LRCW2. Late Roman Coarse Wares, Cooking Wares and Amphorae in the Mediterranean : Archaeology and archaeometry, Oxford, 2007, p. 15-28.

${ }^{26}$ G. FIACCADORI, I frammenti iscritti, in G. Bermond Montanari (ed.), Ravenna e il porto di Classe. Venti anni di ricerche archeologiche a Ravenna e Classe, Imola, 1983, p. 238-241.

${ }^{27}$ G.P. BROGIOLO, Dalla fondazione del monastero al mito di Ansa e Santa Giulia, in G.P. Brogiolo (ed.), Dalla corte regia al monastero di San Salvatore Santa Giulia di Brescia, p. 17-33, part. p. 18-19; J. MITCHELL, The painted decoration of San Salvatore di Brescia in context, Ibidem, p. 169-201.

${ }^{28}$ E. TODOROVA, The Medieval Amphorae (9th to 14th Centuries AD) from the Excavation at Silistra in 2007. (Preliminary report), in C. Tzochev, T. Stoyanov and A. Bozkova (eds.), Production and trade of amphorae in the Black Sea. Acts of the International Round Table (Kiten, 2007), Sofia, 2011, p. 131-143; A. F. FERRAZZOLI, Instrumentum domesticum. Tipologia dei reperti ceramici e aspetti delle produzioni e della circolazione dei materiali, in E. Equini Schneider, Elaiussa Sebaste II: un porto tra Oriente e Occidente, Roma, 2003, p. 649-707, part. p. 699, figs. 144-145.

${ }^{29}$ D. CLAUDE, Der Handel im westlichen Mittelmeer während des Frühmittelalters, AAWG, 144, Göttingen, 1985, p. 149-150.

$3^{\circ} \mathrm{V}$. POPOVIĆ, La descente des Kotrigours, des Slaves et des Avars vers la Mer Egée: le témoignage de l'archéologie, in Comptes rendus. Académie des inscriptions et belles letters, 1978, p. 596-648; D.M. METCALF, Avar and Slav invasions into the Balkan peninsula (c. 575-625), in JRA 4, 1991, p. 140-148.

${ }^{31}$ P. CORSI, La spedizione italiana di Costante II, Bologna, 1983.
} 


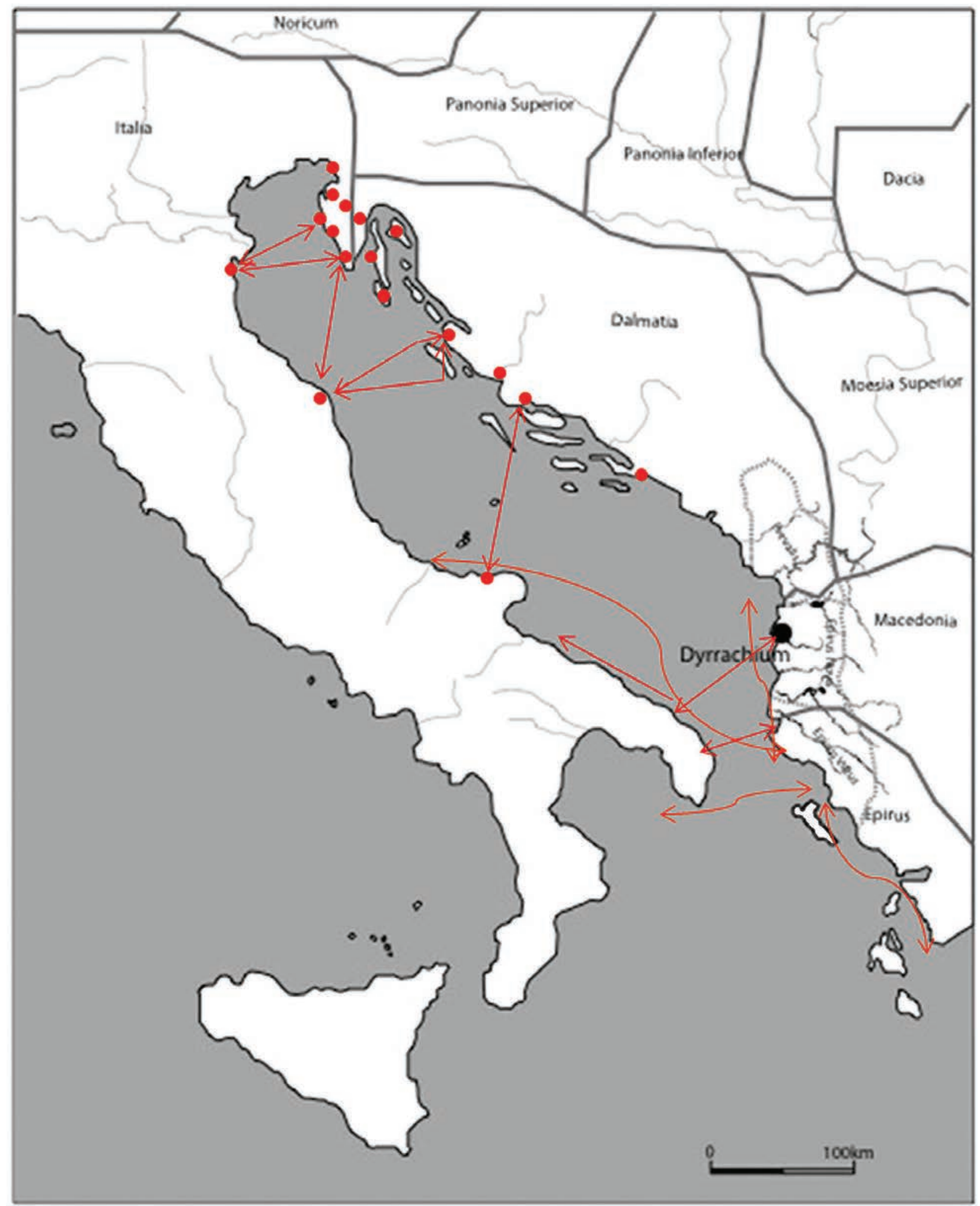

Fig. 1: Carta con indicazione dei principali vettori dell rotte adriatiche.

Non sono molte le tracce lasciate da questa nuova e solo parziale fioritura dei centri costieri, che per il resto mostrano comunque i caratteristici segni di trasformazione e declino delle altre città romane, ma difficilmente vengono abbandonate.

Una crescita degli indicatori che mostrano la vivacità economica di queste zone dell'area ionica sono presenti in diverse zone dell'Epiro settentrionale: Phoinike, Saranda e Butrinto in primis. Per quel che riguarda gli investimenti nell'edilizia disponiamo di importanti contributi da parte dell'équipe inglese sia per quel che riguarda Butrinto ${ }^{32}$ sia per Onkesmos\Saranda. Per Phoinike i nuovi dati mostrano chiaramente la presenza di abitazioni privilegiate nell'area dell'Acropoli, su edifici romani dismessi e la frequentazione

${ }^{32}$ R. HODGES, Byzantine Butrint. Its History and Monuments, London, 2008; P. REYNOLDS, Trade networks of the East, $3 r d$ to 7 th centuries: the view from Beirut (Lebanon) and Butrint (Albania) (fine wares, amphorae and kitchen wares), in S. Menchelli et al. (eds.), LRCW3: Late Roman Coarse Wares, Cooking Wares and Amphorae in the Mediterranean: Archaeology and Archaeometry. Comparison between eastern and western Mediterranean, Oxford 2010, p. 89-114. 
di una vasta area all'interno del circuito ellenistico, sostenuta da un'economia ruruale ancora ricca con grandi insediamenti sparsi, simili a quello documentato nella piana di Vrina ${ }^{33}$. Nel corso degli ultimi anni ho potuto studiare inoltre $\mathrm{i}$ materiali recuperati nell'area dell'Acropoli e in altre zone della città, in gran parte inediti, e abbiamo potuto metterli a confronto con quelli pubblicati negli scavi condotti negli anni '8o dall'Istituto di Archeologia34. La città è inquadrata perfettamente nelle dinamiche degli scambi mediterranei, con una crescita consistente delle quantità di reperti soprattutto nel VI e VII secolo. I principali indicatori sono le forme di Sigillata Africana, in particolare le forme $\mathrm{H}_{105}$, H1o6 e H1o9 ${ }^{35}$. Sono associate a spatheia miniaturistici e a poche anfore grandi cilindriche, in particolar modo del tipo Keay 61. Il maggior numero di anforacei proviene tuttavia dal Mediterrano orientale. Sono rappresentate soprattutto anfore LRA 2 dell'Argolide e le più recenti varianti delle LRA1, sia quelle prodotte nel sud della Turchia, sia quelle di produzione cipriota. In ordine di quantità segue la forma LRA3 e l'anfora di Samo. Dall'area egea provengono anche diversi contenitori del tipo AM273, con varianti anche in questo caso associate ai decenni finali del VI e al pieno VII secolo. Poche sono invece le attestazioni di anfore palestinesi, sia LRA4 sia AM334. Seguono per numero di individui alcune LRA7 e alcune Keay 52, di produzione calabrese, senza lo schiarimento salino sulla superficie esterna. Si segnalano inoltre una decina di esemplari di anfore globulari di VIII secolo, provenienti da alcune abitazioni trovate a ridosso dell'area della basilica, con impasti marnosi, per descrizione simili a quelli rinvenuti in Italia nordadriatica, in particolar modo a Comacchio ${ }^{36}$ e Classe. Se ne trovano però anche esemplari a Cervia, Rimini, Senigallia, Venezia, Padova e Verona. Si tratta di piccoli contenitori provenienti da varie regioni del Mediterraneo, in particolar modo dall'area egeo-anatolica, ma anche dall'Africa del nord e dall'Italia meridionale. Tra il vasellame fine da mensa si segnala anche un gruppo di piatti di forma Hayes 3, di produzione focese, un singolo esemplare attribuibile all'area di produzione egizia e un esemplare di produzione cipriota. Il principale mercato di approvvigionamento di questo materiale è senz'altro Onkesmos\Saranda, dove si riscontrano le stesse qualità di materiali, anche se le quantità sono ancora da definire ${ }^{37}$. L'insediamento, considerato come il vero e proprio sbocco marino della città di Phoinike, divenne un centro urbano con un sistema difensivo autonomo, solo sul finire del V secolo, o secondo alcuni nel corso della metà del VI secolo. Dall'area egea e dalla Palestina provengono anche alcune forme di vasellame da cucina, la cui diffusione in aerea ionica e adriatica è estremamente diffusa ${ }^{38}$. Non sono però numerose, probabilmente a causa della forte concorrenza offerta da produzioni da cucina epirota, il cui mercato sembra avere successo anche nelle sponde dell'Adriatico meridionale. Appartiene probabilmente ad ateliers locali anche la produzione di alcune giare rinvenute in diverse aree della città, spesso associate a contesti di VII secolo, caratterizzate da una decorazione stampigliata sull'orlo e da solcature parallele ondulate o intrecciate, incise a volte con le dita, altre con uno strumento a punta, sia sull'orlo, sia sul corpo dei contenitori. Si tratta di esemplari alti circa un metro, con un diametro dell'orlo compreso tra i 35 e i 40 $\mathrm{cm}$ ca, destinati alla conservazione domestica. Da verificare invece il raggio di diffusione di alcuni contenitori da trasporto, con forma globulare, prodotti anch'essi con impasto epirota, in un periodo compreso tra V e VIII secolo, attestati per ora soprattutto a Phoinike, con un profilo caratteristico e al momento del tutto inedito. I rapporti quantitativi sono esemplificati nel grafico che mostriamo di seguito, senza però soffermarsi troppo sui valori che sono basati al momento solo su un totale di settecento esemplari circa.

Anche se i dati quantitativi per le altre città epirote non sono ancora disponibili, le forme per ora pubblicate sono del tutto simili, come mostrano i repertori di Saranda e quelli studiati per Butrinto, oltre alle raccolte di materiali esposte nelle collezioni dei musei di Igoumenitza e Ioannina, e provenienti da vari siti dell'Epiro greco, tra cui i reperti del porto di Ladicori che ha restituito diversi esemplari di frie pans e di anfore di tipo 'cisterna di Samo', databili tra fine VI e VII secolo39.

Questo quadro di attestazioni è molto differente rispetto a quanto verificato nelle regioni medio e nord-adriatiche. Sono diversi soprattutto i rapporti percentuali tra le diverse produzioni e anche la curva di attestazioni delle quantità nel periodo che va dal IV all'VIII secolo. I contesti finora studiati della città di Classe mostrano ad esempio un picco delle importazioni verso la metà del V secolo e una sostanziale tenuta del volume delle merci fino alla guerra tra Goti e Romani, verso la metà del VI secolo. In questo periodo abbiamo osservato nei siti adriatici, in particolar modo a Ravenna e nel suo territorio una inversione di tendenza delle importazioni dall'Africa settentrionale, verso il Mediterraneo orientale, a dimostrazione del fatto che la guerra di Giustiniano non fu soltanto un'impresa militare, ma anche una conquista del mercato, da parte dei mercanti orientali, nei porti occidentali, una torsione che trova qualche riscontro anche in Tunisia, in particolar modo a Meninx e Cartagine ${ }^{40}$. Il recesso delle importazioni tra VII e VIII secolo è tuttavia

33 E. GIORGI, I. BOGDANI, Il territorio di Phoinike in Caonia. Archeologia del paesaggio in Albania meridionale, Bologna, 2012. Per un aggiornamento sugli scavi si veda: S. DE MARIA, S. GJONGECAJ (eds.), PHOINIKE VI. Rapporto preliminare sulle campagne di scavi e ricerche 2011-2014, Bologna, 2015.

${ }^{34} \mathrm{~K}$. LAKO, Enë balte nga qyteti i Onhezm-Ankiazmit (Saranda')/ Sixth- to seventh-century AD pottery from the city of Onchesmos (Saranda), in Illiria XXX, 2002, p. 283-316.

35 M. MACKENSEN, Production centres of African Red Slip Ware (2nd-3rd c.) in northern and central Tunisia: Archaeological provenance and reference groups based on chemical analysis, in Journal of Roman Archaeology 19, 2006, p. 163-190.

${ }^{36}$ C. NEGRELLI, Dal VI all'VIII secolo: continuità e rotture nella circolazione dei manufatti ceramici tra Romagna e Delta padano, in Le forme della crisi, op cit. (n. 10), p. 139-149.

${ }^{37}$ O. GILKES, R. HODGES, J. VROOM, K. KONDO, New light on early medieval Saranda, ancient Onchesmos: excavations at the Bashkia of Saranda, in Annual of the British School at Athens. Ringrazio gli autori per avermi consentito di visionare il testo ancora in corso di pubblicazione.

${ }^{38}$ M. CAVALAZZI, E. FABBRI, Ceramiche da cucina di V-VII secolo dallo scavo del porto di Classe (RA), in Le forme della crisi, op cit. (n. 10), p. 21-28.

39 A. KANTA-KITSOU, O. PALLI, I. ANAGNOSTOU, Igoumenitsa Archaeological Museum, Igoumenitsa, 2008, p. 39, figg. 4-5 (l'indicazione nella didascalia riporta però una cronologia più alta).

$4^{\circ}$ M. BONIFAY, Africa: patterns of consumption in coastal regions versus inland regions. The ceramic evidence (300-70o A.D.), in L. Lavan (ed.), Local Economies? Production and Exchange of Inland Regions in Late Antiquity, Leiden, Brill, 2013, p. 529-566. 


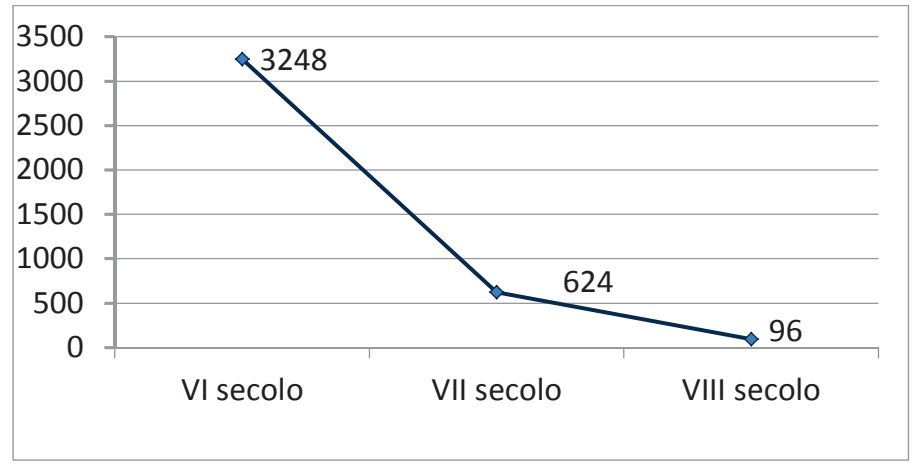

Fig. 2: Numero minimo di esemplari di anfore attestate nell'area portuale di Classe (Ravenna).

piuttosto evidente nell'area del porto di Classe e lo stesso quadro sta emergendo nell'analisi del materiale in corso di studio e in parte pubblicato proveniente dai contesti di Senigallia ${ }^{41}$. La circolazione di merci transmediterranee nel VII e VIII secolo è documentata, ma le quantità di materiali rinvenuto rispetto ai secoli precedenti è in questo periodo molto più modesta (fig. 2).

Il confronto tra queste due regioni di circolazione mediterranea mostrano quindi una diversa composizione e diverse tendenze. Il flusso di commerci che circola verso l'area ionica ${ }^{42}$ segue direttrici non sempre connesse con quelle adriatiche. Dopo il canale di Corfù alcune merci seguono il loro percorso verso il golfo di Taranto, mentre le merci che raggiungono gli scali siciliani, risalgono le coste adriatiche da sud a nord, così come anche dimostrato dalle descrizioni dei viaggi compiuti da Costantinopoli a Ravenna, in occasione ad esempio del ritorno, agli inizi dell'VIII secolo, del vescovo Felice, che dopo essere stato condotto in prigione nel 710, una volta liberato raggiunse prima Siracusa e proseguì il suo percorso lungo le coste ioniche e poi adriatiche. Lo stesso percorso dovevano probabilmente percorrere le merci che seguivano la traiettoria ionica senza approdare sulla sponda orientale, ma con cabotaggio costiero risalivano l'Adriatico. Allo stesso modo le merci che transitavano dalle coste epirote potevano seguire le correnti verso le coste italiane, poco dopo il golfo di Saranda o più a nord all'altezza di Durazzo, oppure risalire le coste orientali dell'Adriatico e raggiungere gli approdi istriani ${ }^{43}$. La predilezione di alcuni tipi di vino rispetto ad altri, anche se provenienti dalle stesse traiettorie commerciali è dimostrata dalla scelta di maggiori quantitativi di anfore di Gaza, così poco rappresentate nei contesti epiroti, mentre nell'area romagnola risultano tra le principali, mentre vi risulta pressoché assente il vino dell'Argolide, contenuto nelle LRA2, quasi invasivo al contrario in questi contesti di area ionica e destinato soprattutto ai contingenti sul limes danubiano ${ }^{44}$. Diverse traiettorie dunque e anche dinamiche differenti.

Questo commercio era attivo su entrambe le coste adriatiche, dall'Epiro e dall'Illiria alla Dalmazia o dall'Apulia verso il nord ${ }^{45}$. Fino alla fine dell'VIII secolo fu in gran parte gestito da mercanti orientali e rimasero sotto il controllo dell'amministrazione bizantina molte città istriane, incluse Trieste, Koper, Novigrad, Rovinj, Pula e Labin, oltre al territorio di Motovun e Buzet ${ }^{46}$. Lo stesso avvenne in Dalmatia, con le isole di Kvarner (Osor and Arbe), Krk, e fino alle coste di Zadar, Trogir e Cattara ${ }^{47}$.

Gli insediamenti di Spalato e Ragusa\Dubrovnik iniziarono ad assumere caratteristiche protourbane in questo periodo, sia come fortificazioni e luogo di rifugio dalla pressione degli Slavi nel territorio, prima di essere trasformati in importanti luoghi di mercato ${ }^{48}$. In alcune zone delle coste dalmate sono state rinvenute alcune evidenze di commercio con il Mediterraneo orientale, soprattutto databili al VII e all'VIII secolo, a Polače-Mljet, a Trilj, vicino Sinj, nel retroterra di Spalato ${ }^{49}$. Se ne trovano tracce inoltre vicino al sito altomedievale del monastero Krka e a Punta Pernat (Cres), in particolar modo preziosi oggetti in metallo, ma solo pochi esemplari di ceramica importata, almeno per il momento. In insediamenti come Lastovo, Kornati, Premuda sono stati anche rinvenuti alcuni relitti che mostrano alcuni cambiamenti nelle imbarcazioni, con la realizzazione di navi più piccole e leggere ${ }^{50}$. Secondo alcuni studiosi il carico di queste imbarcazioni era notevomente cambiato anche nella natura del suo assemblaggio e queste imbarcazioni potevano trasportare contemporanamente: vino, olio e aridi ${ }^{51}$.

Sono inoltre menzionati, nelle fonti scritte, numerosi negotiatores nel principale terminale di questi scambi adriatici ancora nel corso del VII, cioè Ravenna. Basti pensare al negotiator Inga, un mercante attestato in un'epigrafe del $632^{52}$.

${ }^{41}$ F. GALAZZI, I materiali di Senigallia (An) tra Tardoantico e alto Medioevo, in Le forme della crisi (n. 10), p. $291-298$.

${ }^{42} \mathrm{~J}$. VROOM, Early medieval pottery finds from recent excavations at Butrint, Albania, in S. Gelichi (ed.), Atti del IX Congresso Internazionale sulla ceramica medievale nel Mediterraneo (Venezia, 2009), Firenze, 2012, p. 289-296.

43 V. VIDRIH PERKO, Seaborne Trade Routes in the North-East Adriatic and their Connections to the Hinterland in the Late Antiquity, in G.P. Brogiolo, P. Delogu, op. cit. (n. 21), p. 49-77.

44 O. KARAGIORGOU, LR2: a container for the Military annona on the Danubian Border?, in S. Kingsley, M. Decker (eds.), Economy and Exchange in the East Mediterranean during Late Antiquity, Proceedings of the conference at Somerville College (Oxford 1999), Oxford, 2001, p. 129-166.

${ }_{45}$ J. VROOM, From one coast to another: early medieval ceramics in the southern Adriatic region, in S. Gelichi, R. Hodges (eds.), From one sea to another. Trading places in the European and Mediterranean Early Middle Ages, SCISAM 4, Turnhout, 2012, p. 353-391.

${ }_{46}^{6}$ M. VICELJA-MATIJAŠIĆ, Byzantium and Istria: some aspects of byzantine presence in Istria, in Acta Histriae 13 2005 , p. $185-204$.

47 D. DZINO, Becoming Slav, Becoming Croat. Identify Transformations in Post-Roman and Early Medieval Dalmatia, Leiden, 2010, p. 155-175.

${ }^{48}$ F. CURTA, A note on trade and trade centers in the eastern and northern Adriatic region between the eighth and the ninth century, in HAM $16,2010, \mathrm{p}$. 267-276.

${ }_{49}$ M. PETRINEC, Metal Objects of Byzantine Origin in Medieval Graves from Croatia, in P. Grotowski, S. Skrzyniarz (eds.), Towards Rewriting? New Approaches to Byzantine Archaeology and Art, Proceedings of the Symposium on Byzantine Art and Archaeology (Cracow, 20o8), p. 197-212.

$5^{\circ}$ J. FERLUGA, Navigation et commerce dans l'Adriatique au VIIe et VIIIe siècles, in Byzantinische Forschungen 12, 1987, p. 49-51.

${ }^{51}$ A. CARILE, La società ravennate dall'Esarcato agli Ottoni, in Storia di Ravenna, II.2, Dall'età bizantina all'età ottoniana. Ecclesologia, cultura e arte, Venezia, 1992, p. 379-404, part. p. 394.

${ }^{52}$ Corpus Inscriptionum Latinarum XI, 3, 6779 (=Inscriptiones Latinae Christianae Veteres II, 3848); S. COSENTINO, Ricchezza e investimento della chiesa di Ravenna tra la tarda antichità e l'alto medioevo, in S. Gelichi, R. Hodges, op. cit. (n. 42), p. 417-440, part. p. 431. 
Alcuni di questi sono certamente Venetici, e il loro numero cresce nella seconda metà dell'VIII e ancora maggiormente tra IX e X secols3, dimostrando uno sviluppo predominante di mercanti del ducato in confronto a quanto avviene per le altre regioni adriatiche, sebbene il ruolo egemonico di questi gruppi si imporrà solamente nel corso del X secolo ${ }^{54}$. Tra la tarda Antichità e l'alto Medioevo le città adriatiche e in particolar modo Ravenna, non sembrano perdere quel ceto artigiano e commerciale, cresciuto all'ombra delle attività economiche della corte imperiale e poi esarcale, i cui membri nelle fonti scritte altomedievali sono definiti viri honestij5. I mercanti e le nuove forme dell'aristocrazia urbana altomedievale dovettero godere di un certo benessere economico e parteciparono attibamente alla vita economica delle città adriatiche entrando in stretta relazione con le élites militari e vescovili.

Il commercio di Ravenna con le altre città adriatiche sembra limitarsi all'acquisto di formaggi, lana cuoio, pellame, vino, olio, cera e schiavi, come possiamo evincere dal divieto imposto nel 96o, riguardo il commercio di confratelli verso i 'Saraceni', un importante vettore commerciale del mercato di Venezia, Istria e Dalmatia verso l'Italia meridionale ${ }^{56}$.

Conosciamo inoltre altri indicatori di commercio, sia dalle fonti scritte sia da quelle archeologiche. Alcuni chiari indicatori che mostrano un rinnovato impulso produttivo già nel corso dell'VIII secolo, nel territorio romagnolo, a dimostrazione di un tentativo da parte degli arcivescovi ravennati di creare nuove risorse nel proprio territorio, entro i confini del vecchio esarcato, forse in una forma più limitata, tra l'Adriatico e l'Appennino romagnolo\toscano e tra Po e Panaro ${ }^{57}$ così come avevano fatto i Vescovi di Roma nel Lazio, con l'invenzione delle domuscultae ${ }^{58}$.

Lo dimostrano le varie evidenze di alcuni centri del territorio ravennate, in alcune zone della città di Classe, ormai in declino ${ }^{59}$ dove sono state rinvenute tracce di produzione di vasellame di uso domestico e di prodotti vetrari ${ }^{60}$ e nel territorio Decimano, soprattutto in un sito datato alla fine del IX secolo, Montaccio ${ }^{61}$ e all'interno di nuovi centri direzionali del territorio ravennate. Spesso associate a esemplari di ceramica a vetrina pesante. Si tratta di nuovi centri del popolamento, 'central places', ovvero insediamenti rurali dove si osserva la polarizzazione di più antichi insediamenti

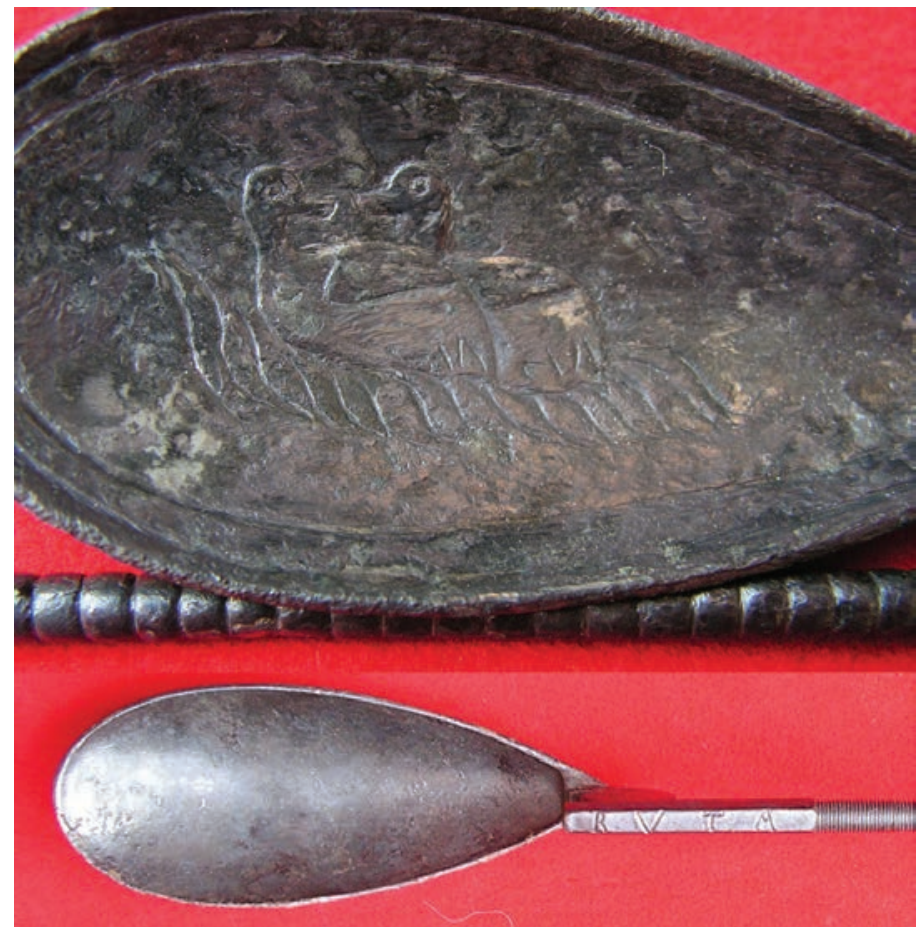

Fig. 3: Particolare di due cucchiai in argento che fanno parte del tesoretto di Classe, con indicazione del nome del proprietario 'Ruta'. Cortesia della Soprintendenza Archeologica dell'Emilia Romagna.

sparsi $^{62}$. Non sono stati al momento rinvenuti in questi siti prodotti del commercio transmediterraneo e neppure quei piccoli contenitori, prodotti in area nordadriatica, forse destinati al trasporto di pesce, identificati invece in un'altra importante città costiera adriatica, quale Senigallia, poco a nord della città portuale di Ancona ${ }^{63}$, in una zona che fino al IX secolo è ancora sotto il controllo fiscale dell'arcivescovo ravennate, insieme a vaste altre zone della Pentapoli e dell'Umbria, dell'Istria delle Venezie e della Sicilia ${ }^{64}$.

I rapporti con mercanti e con il mercato orientale $\backslash$ costantipolitano sono dimostrati anche da altri prodotti come i cucchiai e la patera in argento finemente cesellati, datati tra la fine dell'VIII e il IX secolo, che recano l'iscrizione dell'artigiano in lettere greche (ION/NH/OY), e probabilmente possedute da un esponente del clero di origine germanica, come indica il nome 'Ruta' (fig. 3), su uno dei cucchiai ${ }^{65}$.

\footnotetext{
${ }_{53}$ S. COSENTINO, Antroponimia, politica e società nell'Esarcato in età bizantina e post-bizantina, in J.M. Martin, A. Peters-Custot, V. Prigent (eds.), L'héritage byzantin en Italie (VIIIe-XIIe siècle), II, Les cadres juridiques et sociaux et les institutions publiques, Paris, 2012, p. 173-185, part. p. 183.

54 A. CARILE, La formazione del ducato veneziano, in A. Carile, G. Fedalto (eds.), Le origini di Venezia, Bologna, 1978, p. 1-250.

55 T.S. BROWN, Gentlemen and Officers. Imperial Administration and Aristocratic Power in Byzantine Italy A. D. 554-80o, Hertford, 1984, p. 67-69.

${ }^{56}$ J. HOFFMANN, Die östliche Adriaküste als Hauptnachschubbasis für der venezianischen Sklavenhandel bis zum Ausgang des elften Jahrhunderts, in Vierteljahrschrift für Sozial und Wirtschaftsgeschichte 55, 1968, p. 165-181.

${ }^{57}$ A. CARILE, op. cit., p. 395.

${ }^{58}$ P. DELOGU, The Rebirth of Rome in the Eighth and Ninth Centuries, in R. Hodges, B. Hobley (eds.), The Rebirth of Towns in the West, London, 1988 , p. 33-42. 59 D. FERRERI, Tracce di un quartiere artigianale presso la basilica Petriana a Classe, in Economia e territorio, op. cit. (n. 15), fc.

${ }^{60}$ M. VANDINI, S. ARLETTI, E. CIRELLI, Five Centuries of Mosaic Glass at Saint Severus (Classe - Ravenna), in OCNUS Quaderni della Scuola di Specializzazione in Beni Archeologici, Alma Mater Studiorum - Università di Bologna 22, 2014, p. 69-86.

${ }^{61} \mathrm{M}$. LIBRENTI, Primi dati relativi all'età medievale delle ricognizioni di superficie nel territorio delle Ville Unite, in G. Montevecchi, P. Novara (eds.), In agro decimano. Per un catalogo del patrimonio archeologico del territorio a sud di Ravenna, Ravenna, 2000, p. 211-222, part. p. 214.

${ }^{62}$ C. NEGRELLI, Dalla tarda Antichità all'alto Medioevo (V-IX secolo, in S. Gelichi and C. Negrelli (eds.), A misura d'uomo. Archeologia del territorio cesenate e valutazione dei depositi, Firenze, 2008, p. 237-256, part. p. 251.

${ }^{6}$ G. LEPORE et al., Archeologia urbana a Senigallia III: i nuovi dati dall'area archeologica "La Fenice", FoldEr The Journal of Fasti Online 308, 2014, p. 1-32. ${ }^{64}$ S. COSENTINO, op. cit. (n. 49), p. 419.

${ }_{55}$ M.G. MAIOLI, Classe, quartiere portuale, campagna di scavo 2005, tesoretto di oggetti in argento, in Felix Ravenna. La croce, la spada, la vela; l'alto Adriatico fra Ve VI secolo, Exhibition Catalogue (Ravenna, 2007), Milano, 2007, p. 39-44.
} 

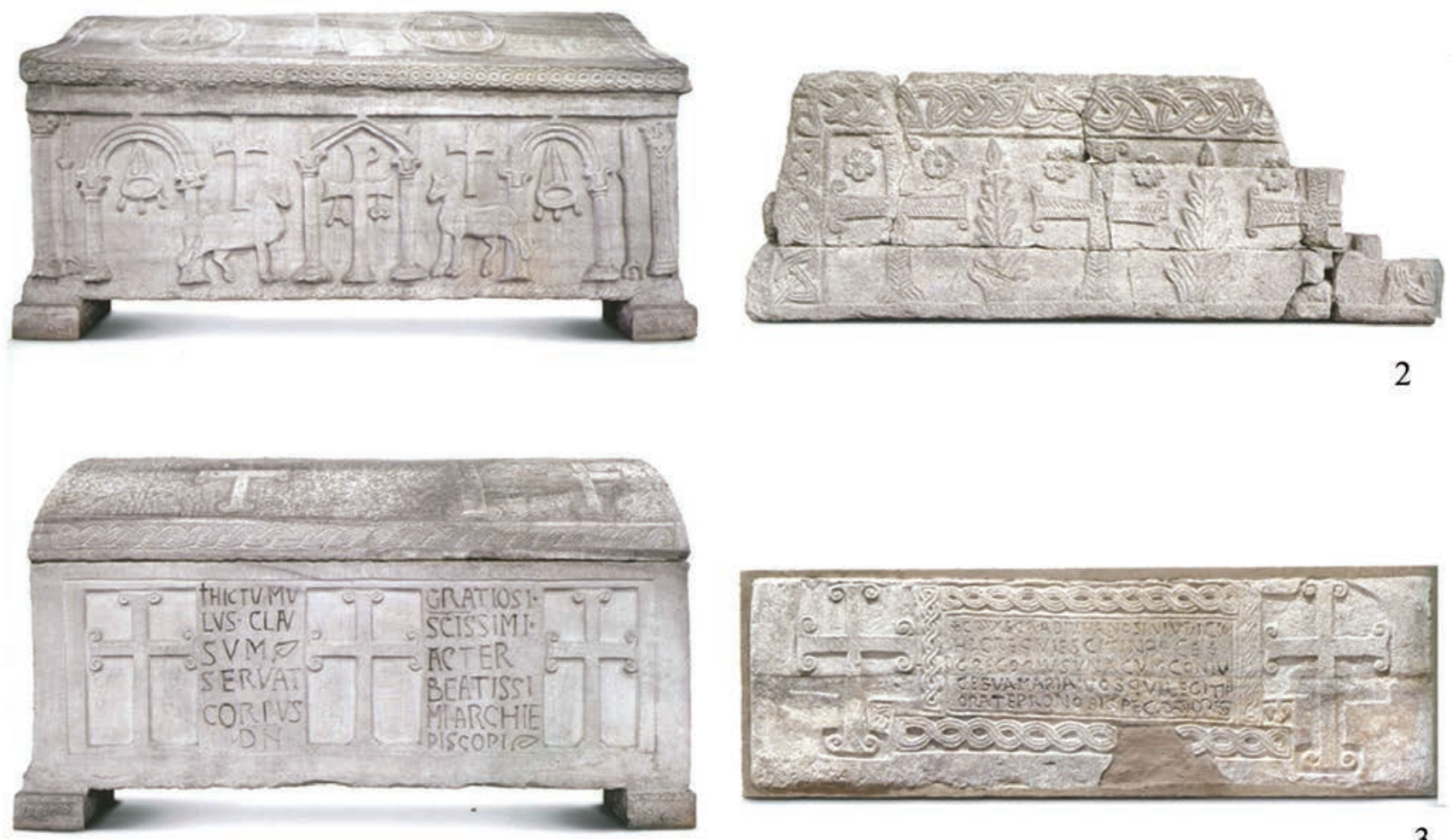

1

Fig. 4: Sarcofagi altomedievali in stile ravennate: 1) Sarcofago di Felix e Gratiosus (VIII sec.); 2) sarcofago rinvenuto nella chiesa di S. Vittore (IX sec.); 3) sarcofago di Gregorius e Maria (IX sec.)

La distribuzione ceramica nelle città della costa adriatica sul versante italiano ${ }^{66}$ e anche nell'hinterland ${ }^{67}$ fino all'estrema punta meridionale, mostrano un notevole dinamismo di questo ceto mercantile ${ }^{68}$. Un altro prodotto di grande successo furono i contenitori in pietra ollare, provenienti dall'area alpina e ampiamente distribuiti lungo le coste adriatiche fino alla Puglia e di seguito fino alla Sicilia, anche tra VIII e IX secolo ${ }^{69}$. Notevoli quantità, databili soprattutto a questo periodo, ne sono state rinvenute a Ravenna e nel suo territorio, in particolar modo nel sito del monastero di San Severo a Classe $^{70}$. Dalle regioni alpine venivano importati anche altri materiali, soprattutto tra VIII eX secolo, le macine circolari, in particolar modo dall'area veneta (Colli Euganei) ${ }^{71}$.

Negli ateliers adriatici, questa volta soprattutto dall'area dalmatina, provenivano anche numerosi oggetti ed elementi

pregiati di arredo liturgico o per residenze delle elites, forse rilavorati da artigiani di area ravennate ${ }^{72}$ e successivamente anche in laboratori istriani come in quello del maestro di Valle $^{73}$. Si tratta di cattedre per i vescovi altoadriatici, come ad esempio quella del vescovo Damiano, prodotta da artigiani ravennati ${ }^{74}$ e anche alcuni altari che appartengono a una nuova tradizione culturale, lontana ormai dai modelli di riferimento 'bizantino'appartenenti ormai a una nuova koiné adriatica ${ }^{75}$. Questi oggetti divennero a loro volta nuovi modelli di riferimento per le élites altomedievali europee, come ad esempio i sarcofagi di IX secolo commissionati dalle aristocrazie altoadriatiche ${ }^{76}$. Ne sono un esempio il sarcofago rinvenuto nella chiesa di S. Vittore a Ravenna, quello del Lapidario Marciano (proveniente dal chiostro di S. Apollonia), quello dei ravennati Gregorius e Maria (fig. 4),

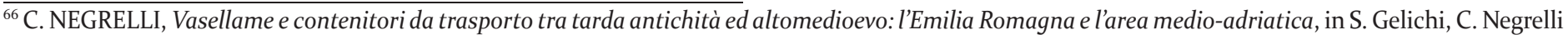
(eds.), La circolazione delle ceramiche nell'Adriatico tra tarda antichità e altomedioevo ( $3^{\circ}$ Incontro di Studio Cer.am.Is sulle ceramiche tardoantiche ed altomedievali, Venezia 2004), edited by, Mantova 2007, pp. 297-330.

${ }^{67}$ H. VERREYKE, P. DE PAEPE, «Late Roman Burnished Ware in Adriatic Italy: The Archaeometric Evidence for Trade from the Central Adriatic Town of Potentia (Porto Recanati), Italy», Journal of field Archaeology, 34 (2009), pp. 185-193.

${ }^{68}$ G. SCRIMA, M. TURCHIANO, «Le ceramiche dei magazzini dell'abitato altomedievale di Faragola (Ascoli Satriano, FG). Tipologie, funzioni e significato sociale», in Atti del VI Congresso Nazionale di Archeologia Medievale, pp. 6o1-606.

${ }^{69}$ R. HODGES, «Adriatic Sea Trade in an European perspective», in From one sea to another, pp. 207-234.

Inserire indicazione anche di Cacciaguerra.

$7^{70}$ M.T. GATTO, La pietra ollare rinvenuta nel sito della basilica di San Severo a Classe, in Economia e Territorio, op cit. (n. 15), fc.

${ }^{71}$ C. PREVIATO et al., Aquileia e le cave delle regioni alto-adriatiche: il caso della trachite euganea, in J. Bonetto, S. Camporeale, A. Pizzo (eds.), Arquelogia de la construcción IV. Las canteras en el mundo antiguo: sistemas de esplotación y procesos productivos, Mérida, 2014, p. 149-166.

${ }_{72}$ Y.A. MARANO, Il commercio del marmo nell'Adriatico tardoantico (IV-VI secolo d.C.), in S. Collodo, G.L. Fontana (eds.), Eredità culturali dell'Adriatico Archeologia, storia, lingua e letteratura, Roma, 2008, p. 159-174.

73 I. MATEJČIĆ, M. JURKOVIĆ, Il lapidario di Valle, Split, 2002, p. 21.

${ }^{74}$ E. RUSSO, Storia e nodi della scultura a Roma nel VI e VII secolo, in Mélanges de l'École Française de Rome. Moyen Âge, Temps Modernes 96, 1984, p. 7-48, part. p. 45 .

75 L. SOTIRA, Gli altari nella scultura e nei mosaici di Ravenna (V-VIII secolo), Bologna, 2013, p. 97.

${ }^{76} \mathrm{G}$. DE FRANCOVICH, Contributi alla scultura ottoniana in Italia: il pluteale di S. Bartolomeo all'isola in Roma, in Bollettino d'arte XXX, 1936, p. $207-224$. 
e quello del vescovo Stefano nella cattedrale di Comacchio, prodotto con calcare Istriano ${ }^{77}$.

Il motore primo del dinamismo economico di questo periodo, venendo meno il peso di una forte fiscalità centralizzata, passa a iniziative di grandi proprietari privati, e in primo luogo, in area adriatica, svolge un ruolo di primo piano l'arcivescovo di Ravenna. La sua ricchezza, costituita da un considerevole patrimonio immobiliare (terre ed edifici), da un tesoro di oggetti preziosi di uso liturgico e da riserve monetarie non differiva sostanzialmente da quella da un grande proprietario laico. Il possesso fondiario era gestito in larga parte attraverso la concessione di terre in enfiteusi o a livello (solo dall'VIII secolo in avanti). Importanti scoperte archeologiche hanno mostrato che si riscontra un cambiamento anche nelle coltivazioni identificate negli scavi romagnoli, dove sono state individuate specie rare in altri contesti europei, come quella del riso, già nel corso dell'VIII secolo ${ }^{78}$.

\section{L'ETÀ CAROLINGIA}

Il periodo di cambiamento più radicale nell'economia della regione adriatica è proprio l'VIII secolo. La fine del governo esarcale privò la chiesa ravennate della vicinanza di un potere politico dal quale era in genere stata sostenuta in molti modi. La ricerca di un nuovo equilibrio con i Franchi, a parte la breve stagione dell'occupazione longobarda, non fu semplice, contrastata com'era dal papato, e mise l'episcopato ravennate di fronte al nuovo interlocutore politico in una posizione di maggiore debolezza rispetto al passato. Ma la ridefinizione dei rapporti politici con il nuovo potere egemone nell'Italia centro-settentrionale avvenne nell'arco di circa mezzo secolo, senza traumi particolari, se si eccettuano episodi eclatanti come l'imprigionamento dell'arcivescovo Giorgio da parte di Carlo il Calvo nell'841.

Un altro dato che sembra emergere con chiarezza nei contesti di VIII secolo di area adriatica è la scarsa o nulla incidenza di casi in cui si possono ipotizzare forme di economia contadina completamente autarchica, con una prevalenza cioè di ceramiche non professionali, modellate a mano e prodotte nell'ambito domestico ${ }^{79}$. Per valutare correttamente sotto il profilo economico la comparsa della ceramica non tornita sembra comunque importante considerarne l'incidenza rispetto ai prodotti artigianali torniti. Nei territori presi in esame le percentuali di ceramiche non professionali non sono mai preponderanti, anche in contesti di VI e VII secolo. Oltre al possibile significato economico (comunità autarchiche che non sono in grado di sostenere con la loro domanda artigiani specializzati e che sono comunque non collegate a reti di scambio sufficientemente efficienti) le ceramiche non tornite possono anche rappresentare un tratto culturale ${ }^{80}$.
Nonostante quindi la riduzione nel numero complessivo delle quantità di prodotti, la commercializzazione di merci è oggi più visibile in questa regione e del resto anche le fonti scritte testimoniano una certa vitalità. Da esse sappiamo ad esempio che l'episcopato possedeva una flotta mercantile, e questa era certamente una delle componenti essenziali per il funzionamento economico del suo patrimonio ${ }^{81}$.

Secondo alcuni studiosi anche i vescovi delle città adriatiche, come gli altri grandi proprietari, reinvestivano una parte delle loro disponibilità nel commercio. Nonostante il divieto della legislazione canonica numerosi chierici si dedicavano alla mercatura come dimostrano molte testimonianze scritte tra VI e IX secolo, tra cui una lettera di Gregorio I del 603, dove viene menzionato il vescovo di Napoli Paschasius indaffarato nei lavori di costruzione di una nave, in cui aveva investito 400 solidi, invece di occuparsi dei poveri ${ }^{82}$.

Anche i vescovi ravennati gestivano le loro proprietà immobiliari ricavandone una fortuna consistente, come indica il Liber Pontificalis e come mostra in precedenza anche l'importante privilegio che Costantino IV concesse al vescovo Reparato e alla chiesa ravennate, come ricorda anche l'importante mosaico rappresentato nella basilica di S. Apollinare in Classe. Si tratta dello stesso tipo di privilegio che agli inizi del IX secolo venne concesso al patriarca di un'altra importante città adriatica, Grado, per quattro navi di proprietà dell'episcopato. Tra le altre cose, i vescovi-imprenditori venivano esentati dal pagamento di alcune imposte sui beni trasporati (teloneum, siliquaticum, laudaticum, cispiaticum).

La visibilità politica del vescovo di Ravenna nell'alto Medioevo costituì un elemento fondamentale nell'imprimere alla città la funzione di volano del commercio in area alto adriatica. I caratteri interregionali del patrimonio e la sua complessità organizzativa caratterizzarono l'economia di questa regione fino ad almeno il IX secolo, soprattutto per i legami con il territorio siciliano nel periodo in cui l'isola venne conquistata dall'emirato aghlabide e sul versante opposto si era già affermato il potere carolingio. La criticità della situazione politica tra VIII e IX secolo mantenne la città in una posizione determinante per le dinamiche del commercio adriatico soprattutto per quanto riguarda la circolazione dell'oro, ancora probabilmente sostenuta dai vescovi ravennati, rendendo il quadro dell'economia monetaria più articolato rispetto al mondo padano.

In area adriatica, come in altre regioni del Mediterraneo, è attestata una grandissima quantità di monete a partire dalla seconda metà del IV secolo, con una progressione verso il VII e una lenta decrescita, fino alle scarsissime attestazioni di VIII secolo (fig. 5).

Questo dato è giustamente messo in relazione con la chiusura della zecca di Ravenna nel 756, che tuttavia non

\footnotetext{
77 R. FARIOLI CAMPANATI, I sarcofagi ravennati con segni cristologici: contributo per un completamento del "Corpus" II, in Felix Ravenna 113-114, 1977, p. 133-159; J. KOLLWITZ, H. HERDEJUERGEN, Die ravennatischen Sarkophage (Die antiken Sarkophagreliefs, 8, 2), Berlin, 1979; S. PATITUCCI UGGERI, Il sarcofago del vescovo-duca Stefano. Contributo alla storia di Comacchio nel sec. IX, in Analecta Pomposiana 5, 1980, p. 7-23.

${ }^{78}$ A. AUGENTI et al., Indagini archeologiche a Classe (scavi 2004): primi risultati sulle fasi di età altomedievale e dati archeobotanici, in R. Francovich, M. Valenti (eds.), Atti del IV Congresso SAMI (S. Galgano, 2005), Firenze, 2005, p. 153-161.

${ }^{79}$ F. CANTINI, Produzioni ceramiche ed economie in Italia centro-settentrionale, in M. Valenti, Ch. Wickham (eds.), Italia 888-962, p. 341-364, part. p. 349-350.

${ }^{80}$ A. MOLINARI, Archeologia e mobilità sociale, in S. Carocci (ed.), La mobilità sociale nel Medioevo, Roma, 2010, p. 117-144.

${ }^{81}$ S. COSENTINO, Constans II, Ravenna's Autocephaly and the Panel of the Privileges in St. Apollinare in Classe: A Reappraisal, in T.G. Kolias, K.G. Pitsakis, C. Synellis (eds.), Aureus. Volume dedicated to Professor Evangelos K. Chrysos, Athens, 2014, p. 153-169.

${ }^{82}$ S. COSENTINO, Credito e finanza a Napoli in una lettera di Gregorio Magno, in R. Shukurov (ed.), Mare et litora. Essays Presented to Sergei Karpov for his $60^{\text {th }}$ Birthday, Moscow, 2009, p. 149-155.
} 


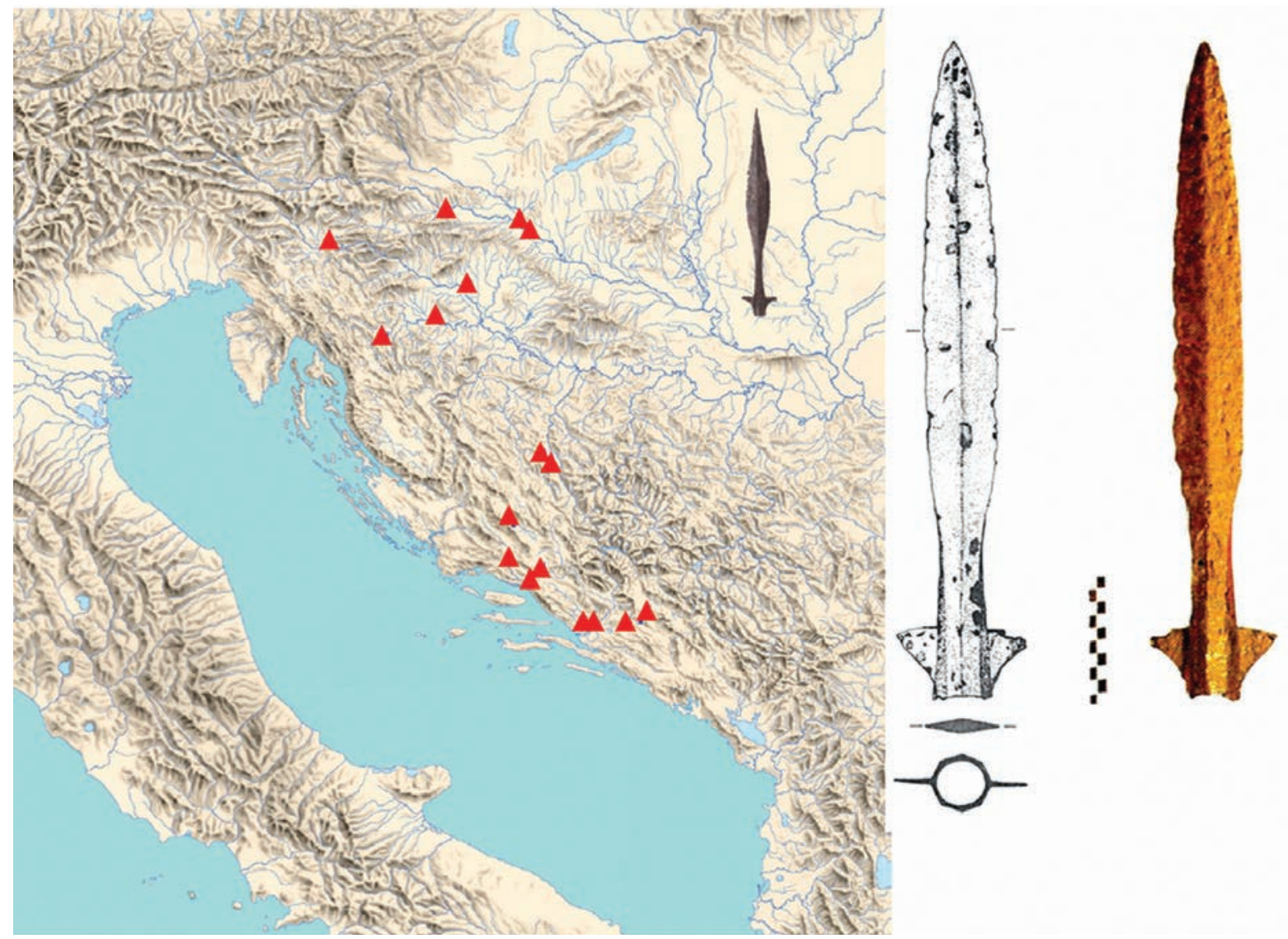

Fig. 6: Carta di distribuzione delle lance ad aletta (rielaborato da TOMIČIĆ 2013)

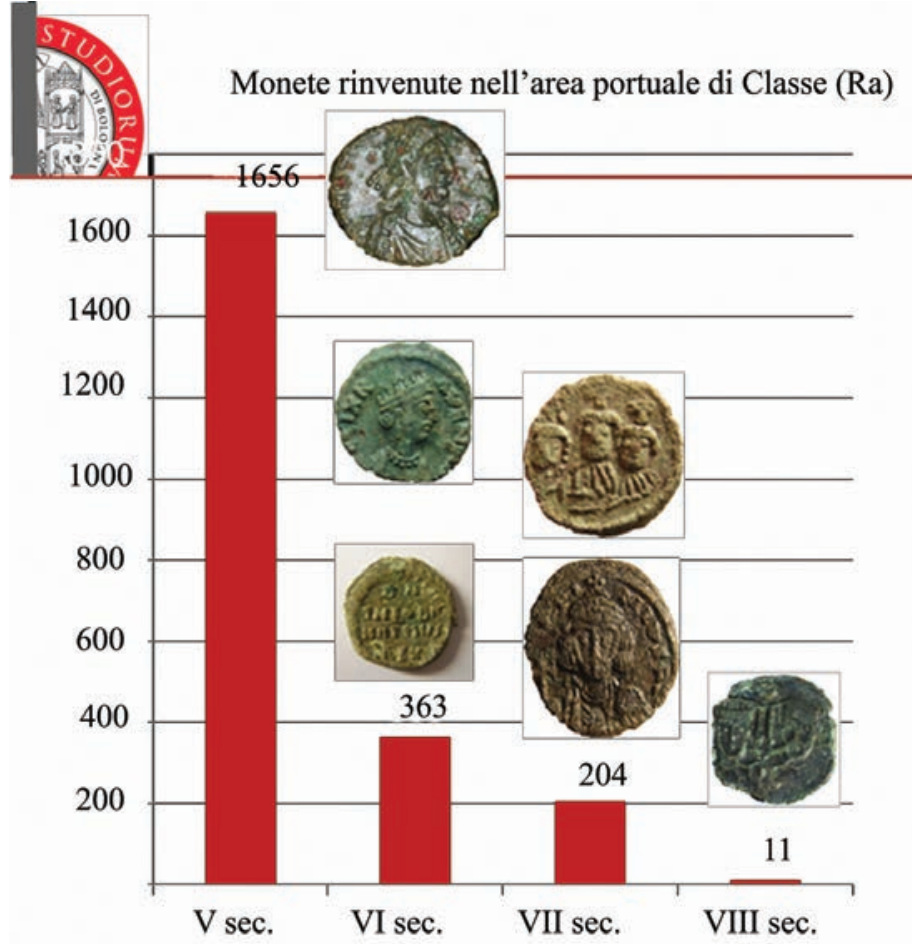

Fig. 5: Grafico delle quantità di monete rinvenute a Classe (elaborato da Elena Baldi). coincise con una totale demonetizzazione dell'area esarcale e pentapolitana. Anche se il denaro carolingio fece fatica a penetrare in questi territori, dove oro e argento circolarono insieme fino alla metà del IX secolo, mi risulta comunque difficile accettare l'idea che l'economia dell'area adriatica sia sostanzialmente tornata a un sistema basato sul baratto o sullo scambio di doni ${ }^{83}$. Vi sono alcune evidenze materiali, come ad esempio le numerose spade carolingie rinvenute in area croata ${ }^{84}$ e le lance con punta ad aletta, rinvenute in diverse zone dell'Italia settentrionale ma anche nella Dalmazia e nella Pannonia ${ }^{85}$ (fig. 6), e finanche reliquari e altre testimonianze di merci pregiate che sono da associare senz'altro a nuove nascenti aristorcrazie ma anche a diverse direttrici commerciali e vie di scambio interne tra l'area renanica e i balcani, distanti dalle tradizionali rotte adriatiche.

Un importante canale di rifornimento della moneta d'oro nei territori nord adriatici, in età carolingia, poté essere rappresentato dall'attività economica della chiesa ravennate, come sottolineato di recente. Fino alla seconda metà del IX secolo (almeno fino all'878) la chiesa di Ravenna continua a immettere in questa regione la moneta d'oro che ricavava dalle proprie rendite in Meridione. Finora sono state trovate, in scavi o in rinvenimenti casuali, 12 monete d'oro (9 solidi e 3 tremisses) e 13 monete di bronzo, nel periodo che va da Leone III (717-741) a Teofilo (828-843), e sono difatti prevalentemente emissioni siracusane ${ }^{86}$.

\footnotetext{
${ }_{83}$ F. CURTA, op cit. (n. 46), p. 273.

${ }^{84}$ G. BILOGRIVIĆ, Carolingian Swords from Croatia - New Thoughts on an Old Topic, in Studia Universitatis Cibiniensis X, 2013, p. 67-83.

${ }_{5}$ Ž. TOMIČIĆ, Karolinško koplje iz varaždina - prinos poznavanju najranije prošlosti grada. In Radovi Zavoda za znanstveni rad HAZU Varaždin 24, 2013., p. 137-152.

${ }^{86}$ S. COSENTINO, op. cit., p. 424.
} 


\section{CONCLUSIONI}

La strada per indagare le trasformazioni delle città adriatiche sono un obiettivo da affrontare a livello materiale sempre più in profondità, cercando di indagare le forme diverse nelle quali la crisi del sistema insediativo di tradizione romana si presentò, dalle pratiche della vita quotidiana alla gestione dei riti funerari ${ }^{87}$, ma senza nascondersi che vi sia stato un grande cambiamento. Fondamentale è chiarire le modalità in cui i diversi territori, in questo caso dell'Adriatico, risposero a queste trasformazioni innescando un fenomeno di 'anticrisi' 88 .

Credo sia infatti importante segnalare gli aspetti di persistenza e di vitalità negli insediamenti urbani, senza identificare meccanicamente il concetto di crisi con quelli di catastrofe, degrado ed estinzione. Questo non implica necessariamente cadere in una debole teoria continuistica. La vita lungo le coste adriatiche continuò senz'altro e le evidenze materiali di questa persistenza sono molte. È necessario però comprendere all'interno di questa continuità le manifestazioni di quei radicali mutamenti delle funzioni e dei rapporti che risultano - alla fine - decisivi per ogni tentativo di ricostruzione storica. La posizione privilegiata delle città adriatiche lungo vettori di commercio marino determinò le fortune dei molti insediamenti che vi si affacciarono ma la contrazione dei volumi degli scambi in questi primi secoli del Medioevo impose ai principali centri di rivolgersi al retroterra produttivo o ai canali di commercializzazione più interni e protetti, senza perdere tuttavia una forte propensione all'attrazione di merci e navi dalle pur rare imprese marittime di questo nuovo Mediterraneo, alle origini della nuova civiltà europea.

${ }_{77}^{8}$ D. FERRERI, Spazi cimiteriali, pratiche funerarie e identitànella città di Classe, in AMXXXVIII, 2011, p. 59-74

${ }^{88}$ C. PAVOLINI, Conclusioni, in Le forme della crisi, op. cit. (n. 10), p. 681-684. 